\title{
Evaluating Immunisation Dropout Rates in Eight Hard to Reach Unions of Maulvibazar District, Bangladesh
}

\author{
Khan Redzwan Habib \\ World Health Organisation, Dhaka, Bangladesh \\ Email address: \\ khan.redz@gmail.com, habibk@who.int \\ To cite this article: \\ Khan Redzwan Habib. Evaluating Immunisation Dropout Rates in Eight Hard to Reach Unions of Maulvibazar District, Bangladesh. \\ International Journal of Immunology. Vol. 5, No. 1, 2017, pp. 5-10. doi: 10.11648/j.iji.20170501.12
}

Received: January 8, 2017; Accepted: January 17, 2017; Published: February 20, 2017

\begin{abstract}
In spite of major achievements in the immunisation sector challenges still remain for the program in Bangladesh, specially to maximize coverage and minimize dropout rates in its Hard to Reach areas. Maulvibazar, a northeastern district of the Bangladesh is one of the 64 districts in the country. In the year 2014 valid vaccination coverage by age of 12 months among 12-23 Month-Old Children in the district for Penta- 1 and for Penta-3 was $89.3 \%$ and $89.6 \%$ respectively and for MR vaccine it was $80.3 \%$. Dropout rate for Penta1-Penta3 vaccines was 3.9\% and Penta1-MR vaccine was $8.7 \%$ in the same year, which is respectively $2 \%$ and $2.9 \%$ higher than national dropout rate. This study aims to determine the dropout rate for immunisation services in Maulvibazar district over a period of one year using Pental to Penta3 and Penta1 to MR vaccine. The study was carried out from January 2015 to December 2015 in eight hard to reach unions of Maulvibazar district over a period of one year. Using the individual union coverage data and immunisation monitoring chart, dropout rates have been calculated among children less than one year of age in the selected unions. When Penta1 and Penta3 vaccine dropout rate is calculated in four months interval it was not statistically significant $(\mathrm{P}=0.267)$. But when Penta1 and MR dropout rate has been calculated in the same period of time it has shown strong significance in dropout rate $(\mathrm{P}=0.012)$. When total number of children receiving Penta1, Penta3 and MR vaccines has been plotted, it has shown a gradual increase in vaccination coverage from January to December. Dropout rates found in this study in selected eight hard to reach unions are very minimal for Penta1-Penta3 vaccine. But for MR vaccine the dropout rates are much alarming. The reason for lower immunisation coverage and higher dropout rates could well be the distance of near by health facilities from these hard to reach areas, bad road conditions and lack of knowledge about vaccination and vaccine preventable diseases.
\end{abstract}

Keywords: Vaccination Program, Immunisation Dropout, Hard to Reach Areas, Hard to Reach Population, Vaccine Preventable Diseases

\section{Introduction}

Bangladesh, officially Peoples' Republic of Bangladesh is a sovereign country in South Asia. It is world's eighth most populated country and fifth most populated country in Asia. Total population of the country as of 2016 is 162,903,941 [1]. The country consists of Eight divisions and sixty-four districts [2]. Immunisation is one of the biggest success stories of the country since its birth in 1971. It is estimated immunisation alone contributes to the reduction of child mortality by $15-20 \%$ in the country [3].

In Bangladesh immunization program is delivered through Directorate General of Health Services (DGHS) with support from World Health Organisation, UNICEF and
Global Alliance for Vaccine and Immunisation (GAVI). EPI or Expanded Program on Immunisation started in Bangladesh on April 7, 1979. As vaccination centres were few and were mainly located in urban areas, EPI coverage remained less than $2 \%$ till 1984 . But it is only after 1985 EPI program was seriously boosted up when the country was committed to United Nations to reach universal child immunisation by 1990 . EPI program was intensified in 476 upazillas, 92 major municipalities and in 6 city corporations [4]. By 1990 immunisation was made available to all target groups. Within 5 years the percentage of fully vaccinated children reached from $2 \%$ in 1986 to $62 \%$ in 1990 . And 
from a crude fully vaccinated rate of $76 \%$ in 1995 the country has achieved an impressive $92.3 \%$ crude fully vaccinated rate by 2014 [5]. Bangladesh along with ten other Southeast Asian countries has been certified as polio free in $27^{\text {th }}$ March, 2014 [6].

Under current EPI schedule in Bangladesh every child under one year receives one dose of Bacille Calmette-Guérin or BCG vaccine, three doses of Pentavalent or Penta vaccine (combination of diphtheria-tetanus-pertussis, Hepatitis B and Haemophilus influenzae type B), four doses of Oral Polio Vaccine or OPV (zero dose at birth), two doses of MeaslesRubella or MR vaccine and three doses of Pneumococcal Conjugate Vaccine or PCV vaccine and one dose of Inactivated Polio Vaccine or IPV [4]. PCV and IPV both vaccines were launch in March 2015 [7]. The EPI program also aims at women of childbearing age and pregnant women for five doses of Tetanus Toxoid or TT vaccine. Government of Bangladesh funds $56 \%$ of all spending on immunisation program [2].

The EPI service delivery mechanism in rural areas relies on 64 districts, 482 upazillas, 4498 unions, 13,494 wards and 108,000 sub-blocks within these wards. Each sub-block has an EPI outreach site where routine immunization services are provided monthly for catchments of approximately 1000 populations. One of the major contributors to this success is Immunisation and vaccine Development (IVD) program of World Health Organisation, Bangladesh. GAVI funds are also supportive of the immunization program. These funds are used for different training, recruiting district level support staffs and sometimes to provide additional support for hard to reach areas or populations [4]. Along with out reach sites vaccination is also performed through fixed sites like Upazilla health complexes (UHCs) and through various non-government organisations. It is the Health Assistants (HAs) who are primarily responsible for immunisation activities with assistance from Family Welfare Assistants (FWAs) [8].

In spite of major achievements in the immunisation program challenges still remain for the program. Only $75 \%$ children of one year of age have received all doses of vaccines, which they are supposed to receive by the first year of life. At the same time significant disparities are seen specially in Penta3 and MR vaccines coverage as 13 out of 64 districts (20\%) has Penta3 coverage less than $80 \%$ and 11 out of 64 districts (17\%) has MR coverage less than $80 \%$ [4]. The total number of children who has received at least one of the recommended vaccines has reached to $81.6 \%$ according to 2014 coverage evaluation survey but this also means still there is $18.4 \%$ children who has never received any vaccine [5].

\section{Current Immunisation Status of the Study Area}

Immunisation coverage varies depending on area and socio-economic groups. While five out of every four children receives at least one dose of the recommended vaccines, only three out of five are being fully vaccinated. Estimated coverage of Penta-3 vaccine was $86 \%$ worldwide and MR vaccines was $85 \%$ worldwide in 2014 , while in Southeast Asia coverage for both vaccines were $84 \%$. In the same year $129(66 \%)$ out of 194 WHO countries have achieved $\geq 90 \%$ national Penta3 coverage and $57(29 \%)$ achieved $\geq 80 \%$ Penta3 coverage in every district [9]. Dropout rates are higher in rural areas specially in hard to reach unions.

Maulvibazar, a northeastern district of the country is one of these 64 districts in Bangladesh. It is a part of Sylhet division. The district is surrounded by Sylhet in the north, Habigonj at the west and Asam and Tripura state of India at east and south respectively. The district's total area is $2799.37 \mathrm{~km}^{2}$ and total population according to 2011 census is $1,919,062$. Every year specially during the rainy season the low lying parts of the district gets flooded by surplus water following through three rivers of the district which have their origin in India. Out of 44 unions of the district, 40 are extremely hard to reach unions (see Picture 1) [10]. Conducting immunisation sessions in these unions are difficult due to their distance and lack of transportation from main land.

According to 2014 coverage evaluation survey, crude vaccination coverage for fully vaccinated child (FVC) by age of 12 months among 12-23 Month-Old Children in the district was $84.1 \%$ and valid coverage was $74.9 \%$. This is consecutively $4.2 \%$ and $6.7 \%$ lower than the national crude and valid coverage. Valid vaccination coverage by age of 12 months among 12-23 Month-Old Children in the district for Penta-1 was $89.3 \%$ and for Penta-3 is $89.6 \%$. Valid vaccination coverage by age of 12 months among 12-23 Month-Old Children for MR vaccine is $80.3 \%$. Dropout rate for Penta1-Penta3 vaccines was $3.9 \%$ and Penta1-MR vaccine was $8.7 \%$ in Maulvibazar district in 2014 which is respectively $2 \%$ and $2.9 \%$ higher than national dropout rate [5].

Therefore, this study aims to determine the dropout rate for routine immunisation program in Maulvibazar district over a period of one year using Penta1 to Penta3 and Penta1 to MR vaccine. This is believed to help the immunisation program to get an idea about and over come a major bottleneck situation in hard to reach areas. At country and local level the study will government and health authorities to improve immunisation activities in areas where coverage is low. Along with this, the study will also form a database based on which further research and policy measures can be carried out. 


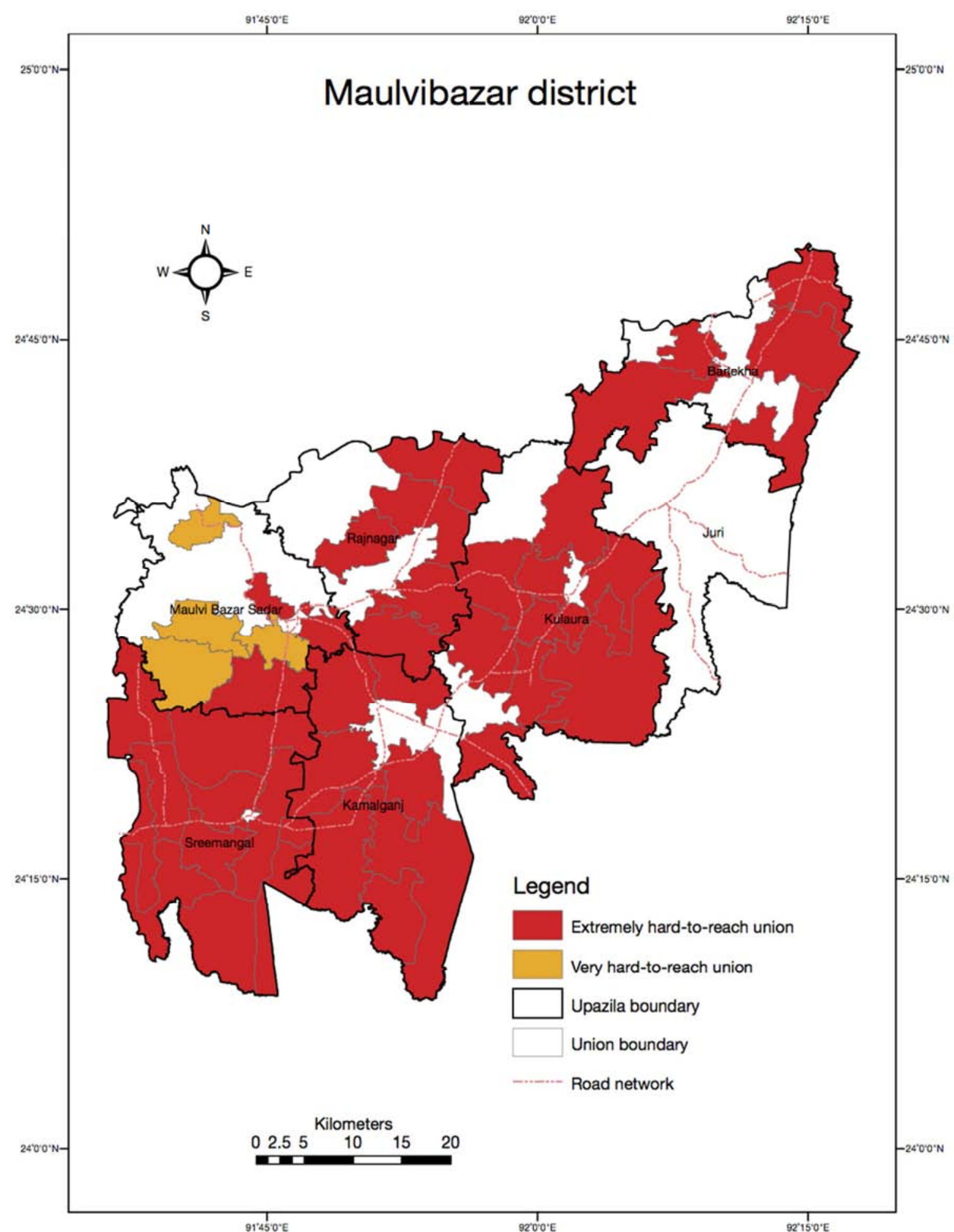

Figure 1. Moulvibazar district map [10].

\section{Method}

The study was carried out in eight hard to reach unions of Maulvibazar district over a period of one year. Total population in these selected unions were 243,526 , total number of 12 months old children were 5435, total number of 12-23 month old children were 4131 and total number of out reach vaccination centres were 788 (see table 1). All eight unions are extremely hard to reach unions. Except for 
Tengra and Kamar Chak six others are hilly regions, Rengra and Kamar Chak are beel (swamp) areas. The out reach centres and upazilla health complexes (UHCs) have data of all eligible children for vaccinations in these areas. Using the individual union coverage data and immunisation monitoring chart, dropout rates have been calculated among children less than one year of age in the selected unions. Children who moved out or moved in within last three months have been excluded. Dropout rates have been calculated using Penta1Penta3 and Penta1-MR.

Table 1. Name and demographics of selected hard to reach unions.

\begin{tabular}{lllll}
\hline Name of hard to reach union & Total Population & $\mathbf{0 - 1 2}$ months old children & $\mathbf{1 2 - 2 3}$ months old children & Total Number of out reach sites \\
\hline Uttar Shahabajpur & 30,631 & 684 & 510 & 98 \\
Uttar Dakshinbhag & 21,934 & 489 & 367 & 82 \\
Patanushar & 32,743 & 731 & 549 & 103 \\
Sagarnal & 36,163 & 807 & 605 & 110 \\
Kadirpur & 29,561 & 660 & 535 & 97 \\
Tengra & 38,539 & 860 & 662 & 114 \\
Kamar Chak & 34,606 & 772 & 579 & 107 \\
Satgoan & 19,349 & 432 & 324 & 77 \\
Total: & 243,526 & 5435 & 4131 & 788 \\
\hline
\end{tabular}

National dropout rate by age of 12 months for Penta1Penta3 is $1.9 \%$ and for Penta $1-M R$ is $5.9 \%$. Dropout rate in Sylhet division is for Penta1-Penta3 is $4.3 \%$ and for Penta1MR is $8.6 \%$. Cumulative dropout rate for Penta1-Penta3 in eight hard to reach unions of this study is $-0.45 \%$ and for Penta1-MR this is $9.8 \%$.

Informed written consent was sought and obtained from respective UH\&FPOs (Upazilla Health and Family planning officer) and ethical approval was taken from Civil Surgeon, Maulvibazar district. Data was analysed using SPSS and Microsoft Excel. Data presentation was in the form of tables and graphs. $\mathrm{P}$ values and Confidence intervals are calculated using Chi-square. The mean and ranges of all variables are calculated. The level of significance was set at $\mathrm{P} \leq 0.05$.

\section{Result}

Total number of children up to 12 months in the selected areas was 5435 in 2015. Among these children 4874 (89.67\%) have received Penta1, $4897(90.10 \%)$ have received Penta3 and $4392(80.80 \%)$ have received MR vaccine. Therefore, the overall dropout rate for Penta1-Penta3 has come up to -23 (Penta1: 4874/Penta3: 4897) and dropout percentage has come up to $-0.46 \%$. Dropout rate and percentage for Penta 1 MR has come up to 482 (Penta1: 4874/ MR: 4392) and 9.80\% respectively (see table 2 ).

Table 2. Immunisation coverage using Penta1-Penta3 and Penta1-MR|Jan, 2015-Dec, 2015.

\begin{tabular}{|c|c|c|c|c|c|c|c|}
\hline Union Name & Penta1 & Penta3 & MR & $\begin{array}{l}\text { Penta1-Penta3 } \\
\text { Dropout Rate }\end{array}$ & $\begin{array}{l}\text { Penta1-Penta3 } \\
\text { Dropout Percentage }\end{array}$ & $\begin{array}{l}\text { Penta1-MR } \\
\text { Dropout Rate } \\
\end{array}$ & $\begin{array}{l}\text { Penta1-MR Dropout } \\
\text { Percentage }\end{array}$ \\
\hline Uttar Shahabajpur & 612 & 614 & 552 & -2 & -0.33 & 60 & 9.8 \\
\hline Uttar Dakshinbhag & 431 & 428 & 394 & 3 & 0.7 & 37 & 8.58 \\
\hline Patanushar & 663 & 667 & 603 & -4 & -0.6 & 60 & 9.05 \\
\hline Sagarnal & 717 & 724 & 648 & -7 & -0.98 & 69 & 9.62 \\
\hline Kadirpur & 586 & 592 & 524 & -6 & -1.02 & 62 & 10.58 \\
\hline Tengra & 790 & 787 & 698 & 3 & 0.38 & 92 & 11.65 \\
\hline Satgoan & 386 & 389 & 348 & -3 & -0.78 & 38 & 9.84 \\
\hline Total & 4874 & 4897 & 4392 & $\approx-3$ & $-0.46 \%$ & $\approx 60$ & $9.80 \%$ \\
\hline
\end{tabular}

Immunisation coverage for 2015 has been stratified into intervals of four months. From January-April 1603 eligible children have received Pental vaccine, 1604 have received Penta 3 and 1460 have received MR vaccine. This gives a dropout rate -1 and percentage $-0.06 \%$ for Penta1-Penta3. Dropout rate and percentage for MR is 91.07 and $9.82 \%$ respectively.

Between May to August 1619 eligible children have received Pental vaccine, 1624 have received Penta3 and 1456 have received MR vaccine. Dropout rate therefore for Penta1-Penta 3 is -5 and percentage is $-0.30 \%$. For Penta $1-$ MR dropout rate is 163 and percentage is $10.06 \%$.

1652 eligible children have received Penta1, 1669 have received Penta3 and MR was given to 1501 children from September to December. Dropout rate and percentage for Penta1-Penta 3 is -17 and $-1.02 \%$ respectively. Penta1-MR dropout rate is 151 and percentage is $9.14 \%$.

Average number of children vaccinated with Pental per month is 406 , Penta3 is 408 and MR is 368 . When Pental and Penta3 vaccine dropout rate is calculated in four months interval it is not statistically significant $(\mathrm{P}=0.267)$ (see table 3 ).

Table 3. Immunisation coverage using Pental and Penta3.

\begin{tabular}{lllll}
\hline & Penta1 & Penta3 & Dropout rate & Dropout \% \\
\hline Jan-Apr & 1603 & 1604 & -1 & $-0.06 \%$ \\
May-Aug & 1619 & 1624 & -5 & $-0.30 \%$ \\
Sep-Dec & 1652 & 1669 & -17 & $-1.02 \%$ \\
Total & 4874 & 4897 & -23 & $-0.47 \%$ \\
\hline
\end{tabular}

But when Penta1 and MR dropout rate has been calculated in the same period of time it has shown strong significance in dropout rate $(\mathrm{P}=0.012)$ (see table 4$)$. 
Table 4. Immunisation coverage using Pental and MR.

\begin{tabular}{lllll}
\hline & Penta1 & MR & Dropout rate & Dropout \% \\
\hline Jan-Apr & 1603 & 1460 & 91 & $9.82 \%$ \\
May-Aug & 1619 & 1456 & 163 & $10.06 \%$ \\
Sep-Dec & 1652 & 1501 & 151 & $9.14 \%$ \\
Total & 4874 & 4417 & 457 & $9.37 \%$ \\
\hline
\end{tabular}

When total number of children receiving Penta1, Penta3 and MR vaccines has been plotted, it shows a gradual increase in vaccination coverage from January to December (see Figure 1).

\section{Total number of vaccinated children}

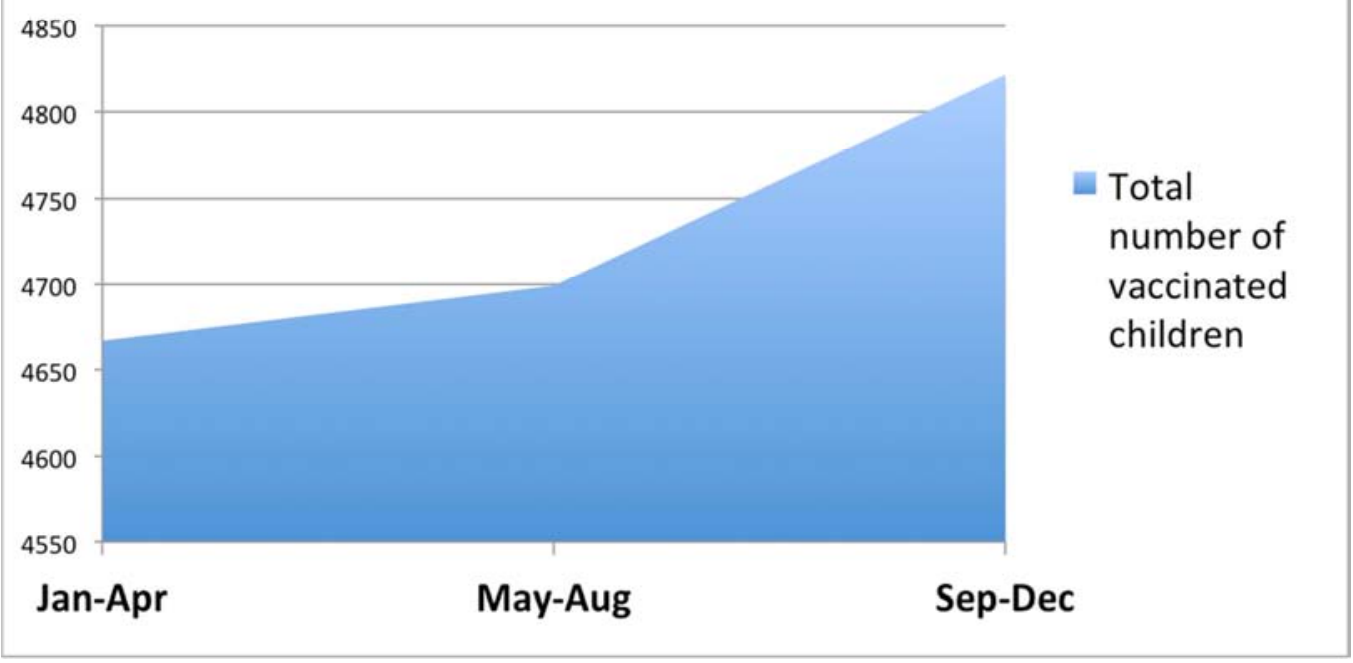

Figure 2. Comparison among vaccination coverage in different parts of the year (January 2015-December 2015).

\section{Discussion}

According to 2014 coverage evaluation survey, comparing to the Sylhet divisional coverage, Maulvibazar district coverage was a bit higher for Penta1 and Penta 3 vaccines $(0.20 \%, 2.25 \%$ respectively); for MR vaccine it was $1.8 \%$ lower than the divisional coverage. When compared to national coverage- valid vaccination coverage by age of 12 months among 12-23 Month-Old Children in the district for Penta-1 was $89.3 \%$, for Penta-3 it was $89.6 \%$ and for MR vaccine it was $80.3 \%$. This is lower than national coverage for Penta 1 by $3.3 \%$, Penta3 by $3.4 \%$ and for MR vaccine by $6.3 \%$. Percentage of fully vaccinated children is also lower in the district than national coverage, $74.9 \%$ against national coverage of $81.6 \%$ [5].

The study has found that in the selected eight hard to reach unions coverage for Pental vaccine from January 2015 to December 2015 was $89.68 \%$, for Penta3 vaccine was $90.10 \%$ and for MR vaccine it was $80.81 \%$. Vaccine dropout rates are also higher in these hard to reach unions comparing to national and other rural areas of the country specially for MR vaccine.

Dropout rates found in this study in selected eight hard to reach unions are very minimal for Penta1-Penta3 vaccine. But for MR vaccine the dropout rates are much alarming. According to World Health Organisation vaccine dropout rate higher than $10 \%$ is not acceptable. This study has found Penta1-MR vaccine dropout rates $9.80 \%$ in selected hard to reach unions, which is therefore very high $[11,12]$.

The reason for lower immunisation coverage and higher dropout rates could well be the distance of near by health facilities from these hard to reach areas. Bad road condition or at times no roads at these hard to reach areas could also play a vital role in their lower vaccination coverage and higher dropout rates. Due to these unions being either hilly or swamp areas continue routine immunisation in the rainy season in these areas could be very much challenging. This has been reflected in the study as well as vaccination coverage being the lowest at the beginning of the year and gradually increasing and reaching at the highest point at the end of the year that is when rainy season ends and winter begins.

Immunisation coverage could be even lower in these hard to reach unions as depicted in the study. The reason behind this is immunisation coverage in rural and hard to reach areas can often "mask" the real situation. According to 2014 coverage evaluation survey about $1 \%$ children have never received any vaccine, which though seem not significant but to eliminate vaccine preventable diseases even one child being left out is not acceptable.

\section{Conclusion}

Immunisation every year save lives of two million children, yet 2.5 million deaths occur every year due to vaccine preventable diseases worldwide [13]. There is scope for further research to explore the reasons for higher dropout and left out rates in rural and hard to reach areas of Bangladesh. The country being a role model for its immunisation achievements needs to identify gaps in its routine 
immunisation program and act accordingly to reach the children in country's hard to reach areas to maximum coverage for vaccine preventable diseases and maintain its polio free status.

\section{References}

[1] Global Alliance for Vaccines and Immunisation, GAVI Country Factsheet: Bangladesh. 2016, Global Alliance for Vaccines and Immunisation.

[2] World Health Organisation, EPI Fact Sheet: Bangladesh 2014 2014, World Health Organisation.

[3] Global Alliance for Vaccines and Immunisation. Application for Country Proposal: Phase 2, Government of the People's Republic of Bangladesh. 2009; Available from: http://webcache.googleusercontent.com/search?q=cache:URx 2SRslSbUJ:www.gavi.org/country/bangladesh/documents/pro posals/proposal-for-nvs---penta-support-bangladesh $/+\& \mathrm{~cd}=1 \& \mathrm{hl}=$ en $\& \mathrm{ct}=\mathrm{clnk} \& \mathrm{gl}=\mathrm{au}$.

[4] Global Alliance for Vaccines and Immunisation, Government of People's Republic of Bangladesh May 2011 Comprehensive Multi-Year Plan. 2011.

[5] Directorate General of Health Services Bangladesh. Bangladesh EPI Coverage Evaluation Survey 2014. 2014 May 11, 2016]; Available from:
http://www.dghs.gov.bd/images/docs/EPI/EPIEvaluationSurve y2014.pdf.

[6] World Health Organisation. WHO South-East Asia Region is officially certified polio-free. 2014; Available from: http://www.searo.who.int/entity/campaigns/poliocertification/en/.

[7] World Health Organisation, Global Immunization News (GIN) 2016, World Health Organisation.

[8] Talukdar, L., et al., The near miracle: how immunization services are delivered. 1991.

[9] Centers for Disease Control and Prevention, Global Routine Vaccination Coverage, 2014. 2014, Centers for Disease Control and Prevention.

[10] World Bank, Hard to Reach Areas: Providing Water Supply and Sanitation Services to All. 2012, World Bank.

[11] World Health Organisation, Communication for polio eradication and routine immunization. 2002, World Health Organisation.

[12] World Health Organisation, The EPI coverage survey. 2008, World Health Organisation.

[13] Burton, A., et al., WHO and UNICEF estimates of national infant immunization coverage: methods and processes. Bulletin of the World Health Organization, 2009. 87 (7): p. 535-541. 